\title{
Rassembler les mots et les choses
}

\section{Christophe Didier}

\section{(2) OpenEdition}

Journals

Édition électronique

URL : http://journals.openedition.org/rbnu/594

DOI : $10.4000 /$ rbnu. 594

ISSN : 2679-6104

\section{Éditeur}

Bibliothèque nationale et universitaire de Strasbourg

\section{Édition imprimée}

Date de publication : 1 novembre 2018

Pagination : 6-7

ISBN : 9782859230784

ISSN : $2109-2761$

\section{Référence électronique}

Christophe Didier, "Rassembler les mots et les choses », La Revue de la BNU [En ligne], 18 | 2018, mis en ligne le 01 juillet 2019, consulté le 10 décembre 2020. URL : http://journals.openedition.org/rbnu/ 594 ; DOI : https://doi.org/10.4000/rbnu.594

\section{(C) $10(0$}

La Revue de la BNU est mise à disposition selon les termes de la Licence Creative Commons Attribution - Pas d'Utilisation Commerciale - Partage dans les Mêmes Conditions 4.0 International. 


\section{Rassembler les mots et les choses}

Une tête babylonienne en albâtre, une brique portant une inscription cunéiforme, des sceaux cylindriques avec leurs empreintes en cire, une tête d'Anubis, une caisse de transport de papyrus sous la forme d'un bidon de pétrole retravaillé, des lampes à huile, une statuette féminine accroupie, un sarcophage de momie de serpent, le fragment de tissu brodé d'un suaire, un scarabée en céramique blanche laquée, un collier égyptien avec une tête de pharaon, une hache de pierre, un moule pour la fonte d'une amulette arabe, un pénis votif en terre cuite issu d'un temple grec d'Égypte, une ficelle ayant servi à serrer un rouleau de papyrus, une bandelette de momie avec inscription, une momie,

des bas-reliefs de Palmyre, une coupe divinatoire juive, une fermeture de sac arabe en bois et corde, un coffret incrusté d'ivoire, un vase de verre à long col, des statuettes moabitiques, dix-neuf pastilles dont certaines portent un caractère, d'autres non, une amulette persane en pierre, des tessères palmyréniennes, un morceau de sel trouvé au bord de la mer Morte, des balles de mousquet en bronze dans une boîte d'allumettes, une sandale de cuir de la fin du $17^{\mathrm{e}}$ siècle, des fragments de silex taillés, une tête de fonctionnaire avec coiffe provenant de Palmyre, une lance de sabre portant des inscriptions, des massues orientales, un tarbouch en tissu clair brodé, un narguilé, cinq chaises persanes refaites, une bague avec intaille en persan " Dieu a la parole et la victoire est proche ", deux stylos à bille à l'effigie de Nizami, trois fragments romains de conduites d'eau en provenance de Carthage, un fragment de meule, un caillou en forme d'œuf, des stèles puniques, une base de colonne avec inscription lihyanite, un fragment de stèle néo-punique avec une fleur de pavot, une hache de Teimâ, le moulage d'un titulus de Malte, un fragment de marbre de l'Acropole d'Athènes, deux poids pour des filets de pêche en provenance de Bari, un fragment de marbre du palais de Tibère sur le Palatin, un gobelet en corne de bovidé, un carreau de faïence ornée provenant de l'Alhambra, une saucière en porcelaine et un plat à gâteau portant les armes et la devise de Gobineau, des coupes en vermeil, un plateau d'argent, une tabatière en or ciselé aux armes de la ville de Colmar, une carafe avec bouchon et monogramme GG, une bonbonnière d'argent, un cendrier, des médaillons représentant Dante,

une bonbonnière de Saxe de la reine Marie de Roumanie, douze assiettes en faïence de Sarreguemines ornées de motifs wagnériens, les lunettes et la serviette de Gobineau, une montre-bracelet de la marque Potonnier, Besançon, des chopes à bière, un olifant en corne de bovidé et en argent, les baguettes de chef d'orchestre d'André Cluytens, Hans Knappertsbusch et Karl Böhm, une broche en poils et brillants représentant une chouette, une patène en argent dédiée à Ernest Wickersheimer par la BNU, une huile sur carton représentant une vieille femme tatare de la Volga, un bas-relief en plâtre peint façon bronze et représentant Tolstoï de profil,

des gravures japonaises, une hache de pierre du Wisconsin, une tête modelée souriante aztèque, un sifflet mexicain, un petit pot aztèque noir, la statue de bois d'un évêque, un buste en terre cuite noir représentant Cicéron, des fragments de statues provenant des façades de la BNU,

le moulage d'une oreille... 
La liste n'est bien évidemment pas close... Tels sont quelques-uns des « objets " que collecte et conserve la Bibliothèque nationale et universitaire de Strasbourg, à côté de ses quelque 3,5 millions de manuscrits et documents imprimés. Ce véritable cabinet de curiosités est-il exceptionnel, dû à la taille et à l'histoire d'un établissement singulier ? Un rapide tour d'horizon d'autres bibliothèques tendrait plutôt à montrer qu'en général, il est rare que celles-ci ne conservent que des livres - quand elles ne sont pas, comme la Bibliothèque nationale de France qui livre dans ce numéro un impressionnant tour d'horizon de ses « collections spécialisées ", d'une certaine façon, de véritables musées.

Cette dimension, longtemps méconnue, ressurgit depuis quelques années, accompagnant une tendance de fond qui a permis, moyennant des rénovations ou à la faveur de nouvelles constructions, la possibilité de donner une nouvelle visibilité aux collections particulières des établissements patrimoniaux. C'est ainsi que la BNU a ouvert au public, en 2014 , ses " réserves visitables " (lesquelles accueillent depuis 2017 un dépôt du... musée du Louvre), que la BnF s'apprête à transformer son site $\mathrm{du}$ quadrilatère Richelieu en « Bibliothèques Musée Galeries », et que d'autres établissements comme la Bibliothèque Humaniste de Sélestat, les Dominicains de Colmar ou l'Inguimbertine de Carpentras (pour ne parler que de la France) affirment clairement désormais leur vocation muséale, à côté de leurs missions traditionnelles qui ne disparaissent pas pour autant.

Il ne s'agit pas ici d'interroger les liens qui unissent - et parfois s'enchevêtrent dans - les notions de bibliothèques et de musées : La Revue s'y attachera dans un prochain numéro. Nous voudrions plutôt rappeler le caractère souvent hétéroclite, et finalement assez universel, de la collecte permanente des bibliothèques, et comment certaines d'entre elles sont confrontées, de façon quasi quotidienne, au contact avec des « objets » - par le biais du dépôt légal (car on édite ou imprime bien autre chose que des pages de papier), par le biais aussi d'achats, de legs ou de donations de fonds composites qu'une démarche scientifique rigoureuse impose de conserver comme autant d'ensembles intellectuellement constitués.

Que l'objet soit, comme instrument de savoir, l'auxiliaire du livre (et vice versa), c'est ce que nous rappelle aussi l'évocation des cabinets de savants de la Renaissance (parents des cabinets de curiosités ou autres « Wunderkammer »), avec l'exemple particulièrement parlant de celui de la cour de Hessen-Butzbach qui convoque les mânes de Kepler, Descartes ou Tycho Brahe. La documentation, dans bien des cas, n'est plei- nement validée que par l'expérimentation - «placet experiri ", ou comment il est nécessaire, parfois, de se confronter à la réalité des choses, et donc de devoir les collecter. N'oublions pas, après tout, que la « documentation » a longtemps été constituée de bien d'autres choses que des livres : tous les objets antiques porteurs d'inscriptions, et plus près de nous, les sceaux et les médailles sont là pour nous le rappeler.

Aujourd'hui toutefois, l'objet fait son entrée en bibliothèque d'une façon plus inattendue, avec une tendance déjà ancienne sur le continent américain, et qui tend à gagner l'Europe depuis quelques années : celle des «bibliothèques d'objets ", où l'on vient pour emprunter une perceuse, une scie électrique, un jouet ou une carte d'entrée dans un zoo. Certains établissements, outre-Atlantique, semblent s'en être fait une spécialité, quand d'autres (à Brest, Bayeux, Paris ou Saint-Étienne) font coexister ces nouvelles « missions sociétales » avec leurs activités de toujours, inaugurant une nouvelle hybridité qui ne laisse pas d'interroger sur le rôle des bibliothèques comme lieux de savoir...

Même si l'on n'est plus là dans une logique de conservation (les objets susmentionnés ne seront gardés que le temps de leur utilisation effective), ni même dans une logique culturelle, mais bien dans celle d'un pur service (et dès lors, le terme de bibliothèque est-il encore pertinent ?), il nous a semblé important de mentionner l'existence (et l'expansion possible) des " grainothèques ", « santothèques " et autres «trucothèques " comme autant d'avatars du biblio-, devenu média- puis fournisseur de services à l'écoute des besoins du plus grand nombre (le fameux « troisième lieu »), avant peut-être de revenir à une réaffirmation de la bibliothèque dans un volet plus strictement documentaire - si nécessaire à une époque de «fake news ", bercée de l'illusion d'une (in)formation immédiatement disponible de chez soi et sans plus d'examen.

La collecte des mots et des choses a une longue tradition; nous souhaitions le rappeler au sein d'un dossier qui prenne le temps d'en montrer toutes les implications, y compris en termes de responsabilité pour les bibliothèques. À l'heure où l'information numérique bouleverse non seulement les pratiques de recherche, mais aussi le rapport aux lieux d'étude et de savoir, il nous a paru important de rappeler la matérialité des supports documentaires qui n'ont cessé d'influencer (et vont, n'en doutons pas, continuer à le faire) les liens que nous entretenons vis-à-vis de la quête du savoir et du rapport à notre patrimoine culturel.

\section{Christophe Didier}

